to Weierstrass, with the totality of those functions $\delta y$ of class $C^{\prime}$ which vanish at $x_{1}$ and $x_{2}$ and satisfy the relation $\delta K=0$. The proof of this lemma - which is an essential step in the chain of conclusions, and whose omission forms a serious gap in the older theory - constitutes the second difficulty.

Neither of these difficulties occurs in the proof which we have given above.

Freiburg, i. B.,

November 19, 1908.

\title{
NOTES ON THE SIMPLEX THEORY OF NUMBERS.
}

BY PROFESSOR R. D. CARMICHAEL.

(Read before the American Mathematical Society, October 31, 1908.)

I. Continued Product of the Terms of an Arithmetical Series.

1. Let $a$ and $c$ be two relatively prime positive integers and form the arithmetical series

$$
x a+c, \quad(x=0,1,2, \cdots, n-1) .
$$

If we inquire what is the highest power of a prime $p$ contained in the product

$$
\prod_{x=0}^{x=n-1}(x a+c), \quad a \neq 0(\bmod p),
$$

we shall find that the general result takes an interesting form. The solution of the problem may be effected in the following manner :

Evidently there exists some number $x$ such that $x a+c$ is divisible by $p$. Let $i$ be the smallest value of $x$ for which this division is possible, and let $c_{1}$ be the quotient thus obtained. Using the notation

$$
H\{y\}
$$

to represent the index of the highest power of $p$ contained in $y$, we will show that

$$
H\left\{\prod_{x=0}^{x=n-1}(x a+c)\right\}=H\left\{\prod_{x=0}^{x=e_{1}}\left(x a+c_{1}\right)\right\}+e_{1}+1,
$$


where

$$
e_{1}=\left[\frac{n-1-i_{1}}{p}\right]
$$

is the largest integer not greater than $\left(n-1-i_{1}\right) / p$. In order to prove (2) we have only to notice that in the product of its first member only factors of the form

$$
\left(m p+i_{1}\right) a+c
$$

contain $p$ and that the quotient of the division is always of the form

$$
m a+c_{1},
$$

and that $e_{1}$ is the highest possible value of $m$. Performing the same operation on the $H$-function of the second member and continuing the process, we should finally arrive at a number which is simply the index of the required power of $p$.

In order to write this result in a convenient form let us define a suitable notation. Let $i_{r}$ be the least integer such that $i_{r} a+c_{r-1}$ contains $p$ and let $c_{r}$ be the quotient of this division. For uniformity, set $c=c_{0}$ and $n-1=e_{0}$. Further, let $e_{r}$ be defined by

$$
\left[\frac{e_{r-1}-i_{r}}{p}\right]=e_{r}
$$

Also let $t$ be the first subscript for which

$$
c_{t}\left(a+c_{t}\right)\left(2 a+c_{t}\right) \cdots\left(e_{t} a+c_{t}\right)
$$

does not contain the factor $p$. Then the preceding result may be written thus

$$
H\left\{\prod_{x=0}^{x=n-1}\left(x a+c_{0}\right)\right\}=\sum_{r=1}^{r=t-1}\left(e_{r}+1\right) .
$$

Since $0 \leqq i_{r} \leqq p-1$, as is evident from the definition of $i_{r}$, we may deduce from (3) the following inequalities:

Hence

$$
\left[\frac{e_{r-1}-(p-1)}{p}\right] \leqq e_{r} \leqq\left[\frac{e_{r-1}}{p}\right]
$$

$$
\left[\frac{e_{r-1}+1}{p}\right] \leqq e_{r}+1 \leqq\left[\frac{e_{r-1}+p}{p}\right] \text {. }
$$


1909.]

This gives

$$
\begin{aligned}
& {\left[\frac{n}{p}\right] \leqq e_{1}+1 \leqq\left[\frac{n-1}{p}\right]+1,} \\
& {\left[\frac{n}{p^{2}}\right] \leqq e_{2}+1 \leqq\left[\frac{n-1}{p^{2}}\right]+1,} \\
& {\left[\frac{n}{p^{3}}\right] \leqq e_{3}+1 \leqq\left[\frac{n-1}{p^{3}}\right]+1,}
\end{aligned}
$$

Taking the sum of these inequalities, we have by (4)

$$
\begin{aligned}
& {\left[\frac{n}{p}\right]+\left[\frac{n}{p^{2}}\right]+\left[\frac{n}{p^{3}}\right]+\cdots \leqq H\left\{\prod_{x=0}^{x=n-1}\left(x a+c_{0}\right)\right\} } \\
& \leqq\left[\frac{n-1}{p}\right]+\left[\frac{n-1}{p^{2}}\right]+\cdots+R(n-1),
\end{aligned}
$$

where $R(n-1)$ is the index of the highest power of $p$ not greater than $n-1$.

This result takes different forms according as $n$ is or is not a power of $p$. If $n$ is a power of $p$, we have evidently

$$
\left[\frac{n}{p^{a}}\right]=\left[\frac{n-1}{p^{\alpha}}\right]+1
$$

for every $p^{\alpha}$ equal to or less than $n$. Remembering that when $n=p^{n}$

$$
\left[\frac{n}{p}\right]+\left[\frac{n}{p^{2}}\right]+\cdots=\frac{p^{h}-1}{p-1}
$$

and using equation (7) in connection with inequality (6), we have

$$
H\left\{\prod_{x=0}^{x=n-1}\left(x a+\mathrm{c}_{0}\right)\right\}=\frac{n-1}{p-1}, \quad n=p^{h} .
$$

When $n$ is not a power of $p$, it is evident that

Suppose now that

$$
\left[\frac{n}{p^{a}}\right]=\left[\frac{n-1}{p^{a}}\right] \text {. }
$$

$$
n=\delta_{h} p^{h}+\delta_{h-1} p^{h-1}+\cdots+\delta_{1} p+\delta_{0}, \quad \delta_{h} \neq 0,
$$

and at least one other $\delta$ is not zero. Employing (9) and the 
well-known formula

$$
\left[\frac{n}{p}\right]+\left[\frac{n}{p^{2}}\right]+\cdots=\frac{n-\left(\delta_{h}+\delta_{h-1}+\cdots+\delta_{1}+\delta_{0}\right)}{p-1},
$$

we may write (6) as follows :

$$
\begin{aligned}
\frac{n-\left(\delta_{h}+\cdots+\delta_{1}+\delta_{0}\right)}{p-1} & \leqq H\left\{\prod_{x=0}^{x=n-1}\left(x a+c_{0}\right)\right\} \\
& \leqq h+\frac{n-\left(\delta_{h}+\cdots+\delta_{1}+\delta_{0}\right)}{p-1} .
\end{aligned}
$$

The inequalities in (11) confine the value of $H$ in narrow limits which are easily calculated.

2. In the series $x a+c$, it may happen that the first $x$ for which $x a+c$ is divisible by $p$ will give $c$ as the quotient of this division. Then in the preceding discussion all the $c$ 's are equal; and then also all the $i$ 's. Dropping subscripts from $i$ and $c$ and making repeated use of equation (3), we have

$$
\begin{aligned}
& e_{1}=\left[\frac{n-1-i}{p}\right], \\
& e_{2}=\left[\frac{e_{1}-i}{p}\right]=\left[\frac{e_{1} p-i p}{p^{2}}\right]=\left[\frac{n-1-i-i p}{p^{2}}\right], \\
& e_{3}=\left[\frac{e_{2}-1}{p}\right]=\left[\frac{e_{2} p^{2}-i p^{2}}{p^{3}}\right]=\left[\frac{n-1-i-i p-i p^{2}}{p^{3}}\right],
\end{aligned}
$$

If we add one to each member of each of these equations and take the sum of the results; then further, if we replace the resulting first member by its value taken from (4), we have

$$
\begin{aligned}
& H\left\{\prod_{x=0}^{x=n-1}(x a+c)\right\}=\left[\frac{n-1-i+p}{p}\right] \\
& \quad+\left[\frac{n-1-i-i p+p^{2}}{p^{2}}\right]+\left[\frac{n-1-i-i p-i p^{2}+p^{3}}{p^{3}}\right]+\cdots
\end{aligned}
$$

3. If $a=c=1$, equation (12) takes a very simple form. For this case $i=p-1$. The result is the well-known theorem that the highest power of $p$ contained in $n$ ! is that whose index is

$$
\left[\frac{n}{p}\right]+\left[\frac{n}{p^{2}}\right]+\cdots=\frac{n-\left(s_{h}+\cdots+s_{1}+s_{0}\right)}{p-1}
$$


where

$$
n=s_{h} p^{h}+s_{h-1} p^{h-1}+\cdots+s_{1} p+s_{0} .
$$

4. If $a=2$ and $c=1$, equation (12) takes a special form of considerable interest. The terms of $x a+c$ are the natural odd numbers in order, and $p$ is an odd prime. It is evident that $i=\frac{1}{2}(p-1)$. Therefore

$$
\begin{aligned}
& {\left[\frac{n-1-i-i p \cdots-i p^{\beta-1}+p^{\beta}}{p^{\beta}}\right]} \\
& \quad=\left[\frac{2 n-2-2 i-2 i p-\cdots-2 i p^{\beta-1}+2 p^{\beta}}{2 p^{\beta}}\right]=\left[\frac{2 n-1+p^{\beta}}{2 p^{\beta}}\right] .
\end{aligned}
$$

Then (12) becomes

$$
\begin{aligned}
& H\{1 \cdot 3 \cdot 5 \cdots(2 n-1)\}=\left[\frac{2 n-1+p}{2 p}\right] \\
& +\left[\frac{2 n-1+p^{2}}{2 p^{2}}\right]+\left[\frac{2 n-1+p^{3}}{p^{3}}\right]+\cdots
\end{aligned}
$$

II. An Extension of Fermat's Theorem.

It will be shown that the congruence

$$
x^{\phi(n)} \equiv 1(\bmod n),
$$

where $\phi(n)$ is Euler's $\phi$-function of $n$, is still true when the modulus is a multiple of $n$ formed in a definite way, $x$ being prime to the new modulus.

It has been shown * that $\phi(z)=a$ has always more than one solution. If $z_{1}$ and $z_{2}$ are two roots of $\phi(z)=a$, then $z_{1}$ and $z_{2}$ must each have a factor not common to the two except when one is an odd number and the other is twice that odd number; and hence, except in this case, their lowest common multiple is greater than either of them. Now if $z_{1}, z_{2}, \ldots, z_{i}$ are all the roots of $\phi(z)=a$, we have by Fermat's theorem the congruences

$$
x^{a} \equiv 1\left(\bmod z_{1}\right), x^{a} \equiv 1\left(\bmod z_{2}\right), \cdots, x^{a} \equiv 1\left(\bmod z_{i}\right),
$$

where in each case $x$ is prime to the modulus involved. Now if $L$ is the lowest common multiple of $z_{1}, z_{2}, \ldots, z_{i}$ and $x$ is prime to $L$, we have

$$
x^{a} \equiv 1(\bmod L),
$$

where $L$ is greater than any number whose totient is $a$ except

* Carmichael, Bulletin, vol. 13, p. 241. 
when the equation $\phi(z)=a$ has only the two solutions $z=L, z=\frac{1}{2} L$. Hence,

THEOREM. Except when $n$ and $\frac{1}{2} n$ are the only numbers whose totient is the same as that of $n$, the congruence $x^{\phi(n)} \equiv 1$ holds for a modulus which is some multiple of $n$.

A working method for finding such a modulus is the following :

Set $\phi(n)=a$, for convenience. Separate $a$ into its prime factors and find the highest power of each prime $p$ contained in $a$ such that $\phi\left(p^{a}\right)$ is equal to or is a factor of $a$. Suppose that the following primes are found $: p_{1}^{\alpha_{1}}, p_{2}^{a_{2}}, \cdots, p_{j}^{a_{j}}$. Then write out all the divisors of $a$ and take every prime $q$ such that $q-1$ is equal to any one of these divisors, but $q \neq$ any $p$; and say we have $q_{1}, q_{2}, \cdots, q_{k}$. Then set

Then evidently

$$
M=p_{1}^{\alpha_{1}} p_{2}^{a_{2}} \cdots p_{j}^{a_{j}} q_{1} q_{2} \cdots q_{k} \text {. }
$$

$$
X^{a} \equiv 1(\bmod M),
$$

when $X$ is prime to $M$. (It should be noticed that $M$ may be a multiple of $L$ in congruence (1).)

As thus defined, $M$ is a definite function of $a$; say $M=M(a)$. For every odd value of $a$, except $a=1$, we have $M(a)=1$, as the reader may readily verify. Some even values of $a$ give also $M(a)=1$. There follows a table giving the value of

\begin{tabular}{|c|c|c|c|c|c|}
\hline$a$ & $M(a)$ & $a$ & $M(a)$ & $a$ & $M(a)$ \\
\hline 2 & 12 & 48 & 2227680 & 104 & 12720 \\
\hline 4 & 120 & 52 & 6360 & 106 & 1284 \\
\hline 6 & 252 & 54 & 43092 & 108 & $\begin{array}{llll}22 & 265 & 704 & 680\end{array}$ \\
\hline 8 & 240 & 56 & 6960 & 110 & 33396 \\
\hline 10 & 132 & 58 & 708 & 112 & $\begin{array}{llll}26 & 740 & 320\end{array}$ \\
\hline 12 & 32760 & 60 & $\begin{array}{lll}3 & 407 & 203800\end{array}$ & 116 & 7080 \\
\hline 16 & 8160 & 64 & 32640 & 120 & $\begin{array}{llll}279 & 390711600\end{array}$ \\
\hline 18 & 14364 & 66 & $\begin{array}{lll}388 & 332\end{array}$ & 126 & $\begin{array}{lll}549 & 092628\end{array}$ \\
\hline 20 & 6600 & 70 & 9372 & 128 & 65280 \\
\hline 22 & 276 & 72 & $\begin{array}{llll}10 & 087 & 262 & 640\end{array}$ & 130 & 17292 \\
\hline 24 & 65520 & 78 & 948 & 132 & $\begin{array}{llll}50 & 483 & 160\end{array}$ \\
\hline 28 & 3480 & 80 & 18400800 & 136 & 10960 \\
\hline 30 & 85932 & 82 & 996 & 138 & 1646316 \\
\hline 32 & 16320 & 84 & $\begin{array}{llll}285 & 962 & 040\end{array}$ & 140 & 13589400 \\
\hline 36 & $\begin{array}{lll}69 & 090 & 840\end{array}$ & 88 & $491 \quad 280$ & 144 & $\begin{array}{llll}342966 & 929 & 760\end{array}$ \\
\hline 40 & 108240 & 92 & 5640 & 148 & 17880 \\
\hline 42 & 75852 & 96 & $\begin{array}{lll}432 & 169 & 920\end{array}$ & 150 & 12975732 \\
\hline 44 & 2760 & 100 & 3333000 & & \\
\hline 46 & 564 & 102 & 25956 & & \\
\hline
\end{tabular}
$M(a)$ for each $a$ for which $M \neq 1$ up to $a=150$. 
III. The Solutions of $\phi(z)=a$.

It is desirable to have a general method for finding all the solutions of

$$
\phi(z)=a
$$

for any given $a$. The method used in Note II for finding $M$ in congruence (1) is suggestive, and we may formulate a rule thus :

Find $M$ as in Note II. Evidently, the solutions of $\phi(z)=a$ will all be factors of $M$. Then examine all the factors of $M$ and retain each one whose totient is $a$.

alabama Presbyterian College, AnNiston, Alabama.

\section{THE SOLUTION OF BOUNDARY PROBLEMS OF LINEAR DIFFERENTIAL EQUATIONS OF ODD ORDER.}

BY PROFESSOR W. D. A. WESTFALL.

E. ScHmidT ${ }^{1}$ has studied the set of linear integral equations with non-symmetric matrix

(1) $\phi_{i}(s)=\lambda_{i} \int_{a}^{\triangleright b} K(s, t) \psi_{i}(t) d t, \quad \psi_{i}(s)=\lambda_{i} \int_{a}^{b} K(t, s) \phi_{i}(t) d t$ and has shown that, if there can be found for a function $f(x)$ a continuous function $h(x)$, such that

$$
f(x)=\int_{a}^{b} K(x, t) h(t) d t
$$

then

$$
f(x)=\sum_{i} \frac{\phi_{i}(x)}{\lambda_{i}} \int_{a}^{b} h(t) \psi_{i}(t) d t,
$$

where $\phi_{i}$ runs over a complete set of solutions of (1) which have been normalized and orthogonalized, $i$. e.,

$$
\int_{a}^{b} \phi_{i} \psi_{j} d x=\left\{\begin{array}{ll}
1, & i=j \\
0, & i \neq j
\end{array}\right\}
$$

* Math. Annalen, vol. 63, p. 459. 